Military Technical College Kobry El-Kobbah, Cairo, Egypt.

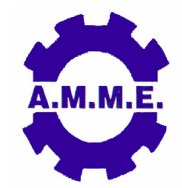
$15^{\text {th }}$ International Conference
on Applied Mechanics and
Mechanical Engineering.

\title{
TRACKING CONTROL OF AN UNDERACTUATED AUTONOMOUS SHIP
}

\author{
S. Samaali* and A. Abichou**
}

\begin{abstract}
In this paper, we will construct a control that forces position and orientation of the underactuated autonomous ship moves according to a reference feasible trajectory. To achieve this objective, we use as a design tool of puts the Backtepping methodology and Lyapunov function. Experimental results are given to show the tracking performance. We will illustrate trajectories with time varying velocity (sinusoidal path). Then, we will test the tracking robustness in presence of drag forces disturbances.
\end{abstract}

\section{KEY WORDS}

Underactuated Ship, Tracking Control, Backstepping Method, Lyapunov Theorem.

Associate Professor, Laboratory of Mathematical Engineering (LIM), Tunisia Polytechnic School, BP 743, 2078 La Marsa, Tunisia, sarrasam@hotmail.fr.

** Professor, Laboratory of Mathematical Engineering (LIM), Tunisia Polytechnic School, BP 743, 2078 La Marsa, Tunisia, Azgal.Abichou@ept.rnu.tn. 


\section{INTRODUCTION}

The past few decades, control of robotic marine vehicles for autonomous navigation has become an intense research area. The interest in the field is widely motivated by the emerging applications such as defense and patrolling of coastal perimeters, naval system applications, harbor operations, marine biology,..etc.

Besides their various missions, marine vessels raise some challenges in control systems theory, because their dynamics often fall in the class of underactuated systems (i.e. Systems where the control vector has lower dimension than the configuration vector). This configuration is by far most common among the ships.

Several control approaches have been presented in the literature to solve the trajectory tracking control problems that require the design of control laws that force the vehicle to track a time parameterized reference (trajectory). Do and Pan [1] addressed the tracking problem of ships that are not actuated in the sway direction and the mass and damping matrices are not assumed to be diagonal and they used the Backstepping technique to design a controller that forces ships to globally track a reference trajectory. Repoulias and Papadopoulos [2], and Santhakumar and Aoskan [3] presented a tracking control algorithm for underactuated autonomous underwater vehicles (AUVs) moving on the horizontal plane (constant depth motion). But in (Repoulias and Papadopoulos [2]), the mass and damping matrices of the AUVs were assumed to be diagonal. However Santhakumar and Aoskan [3] studied the AUVs with non-zero off diagonal terms in the system matrices. In (Lefeber et. al. [4], Pettersen and Egeland [5] Pettersen and Nijmeijer [6], Pettersen and Nijmeijer [7] ), the yaw velocity was required to be nonzero. This restrictive assumption implies that a straight line cannot be tracked. Moreover, Do et. al. [8] succeed to remove the requirement that yaw velocity be nonzero.

Motivated by the variety approaches that have been proposed to solve the trajectory tracking control problem for underactuated autonomous vehicles, we propose in this paper the combined problem of trajectory planning and designing a controller to track a reference trajectory of a ship Repoulias and Papadopoulos [2]. The Backstepping technique of Krstic et. al. [9], that is recursive design methodology for non linear feedback control in order to develop control algorithm, is used. The present paper is composed of 5 sections: In section 2 the equations that describe the ship motion are recalled. Then, in section 3 the tracking control law is developed after planning the reference trajectories. The experimental results are presented in section 4 and in section 5 , conclusion is drawn.

\section{THE SHIP MODEL}

Figure 1 presents the two coordinate frames which we consider in our study, where $x_{1} y_{1} z_{1}$ the Ship-fixed reference and the origin $O_{1}$ is the center of gravity. $x y z$ is the Earth-fixed reference frame. We consider an underactuated ship, that has two controls which provide surge force and yaw moment. The motion matrix model of the ship is described as Pettersen and Egeland [5]: 


$$
\left\{\begin{array}{l}
M \dot{v}+C v+D v=\tau \\
\dot{\eta}=J(\eta) \tau
\end{array}\right.
$$

where:

- $\eta=[x, y, z]^{T}$ is the configuration vector that denotes position and orientation in earthfixed coordinates,

- $v=[u, v, r]^{T}$ is the velocity vector, where $u$ and $v$ are the linear velocities respectively in surge and sway, and $r$ is the angular velocity in yaw,

- $J(\eta)$ is the matrix that describes the ship rotations in the $\left(x_{1} O y_{1}\right)$ plan around $\left(O z_{1}\right)$ axe and it has full rank where its expression is presented by:

$$
J(\eta)=\left[\begin{array}{ccc}
c_{\psi} & -s_{\psi} & 0 \\
s_{\psi} & c_{\psi} & 0 \\
0 & 0 & 0
\end{array}\right]
$$

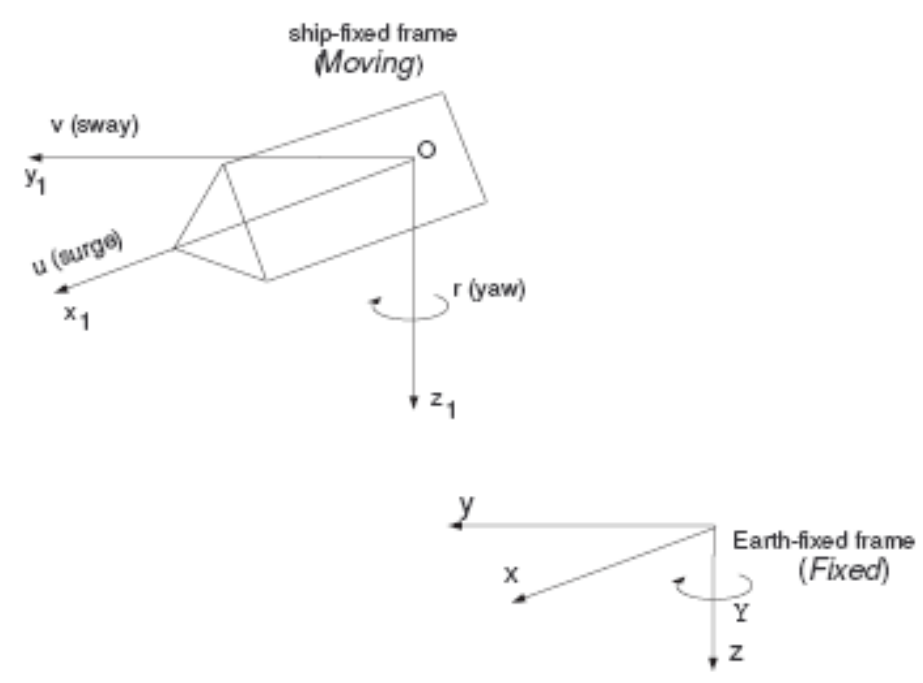

Fig.1. Ship-fixed frame and earth-fixed frame.

Then, the equations describing dynamic characteristics of the ship were derived from Newtonian dynamics laws, with

$$
M=\left[\begin{array}{ccc}
m_{11} & 0 & 0 \\
0 & m_{22} & 0 \\
0 & 0 & m_{33}
\end{array}\right], C=\left[\begin{array}{ccc}
0 & 0 & -m_{22} v \\
0 & 0 & m_{11} u \\
m_{22} v & -m_{11} u & 0
\end{array}\right], D=\left[\begin{array}{ccc}
d_{11} & 0 & 0 \\
0 & d_{22} & 0 \\
0 & 0 & d_{33}
\end{array}\right]
$$

where $M$ is the inertia matrix, $D$ is the damping matrix, $C$ represents centrifugal and Coriolis effects. The matrices $M$ and $D$ are constant and positive definite.

- $\tau=\left[\tau_{u}, 0, \tau_{r}\right]^{T}, \tau_{u}$ is control force in surge and $\tau_{r}$ is control moment in yaw.

Therefore, the developed complete mathematical model of the ship is expressed as: 


$$
\left\{\begin{array}{l}
\dot{x}=u c_{\psi}-v s_{\psi} \\
\dot{y}=u s_{\psi}+v c_{\psi} \\
\dot{\psi}=r \\
\dot{u}=\frac{1}{m_{11}}\left[m_{22} v r-d_{11} u+\tau_{u}\right] \\
\dot{v}=\frac{-1}{m_{22}}\left[d_{22} v+m_{11} u r\right] \\
\dot{r}=\frac{1}{m_{33}}\left[\left(m_{11}-m_{22}\right) v u-d_{33} r+\tau_{r}\right]
\end{array}\right.
$$

The next section is composed of two parts. In the first part, we will focus on planning the reference trajectory Repoulias and Papadopoulos [2] and in the second part we will use the recursive Backstepping controller design that allows us to guide the ship on the course.

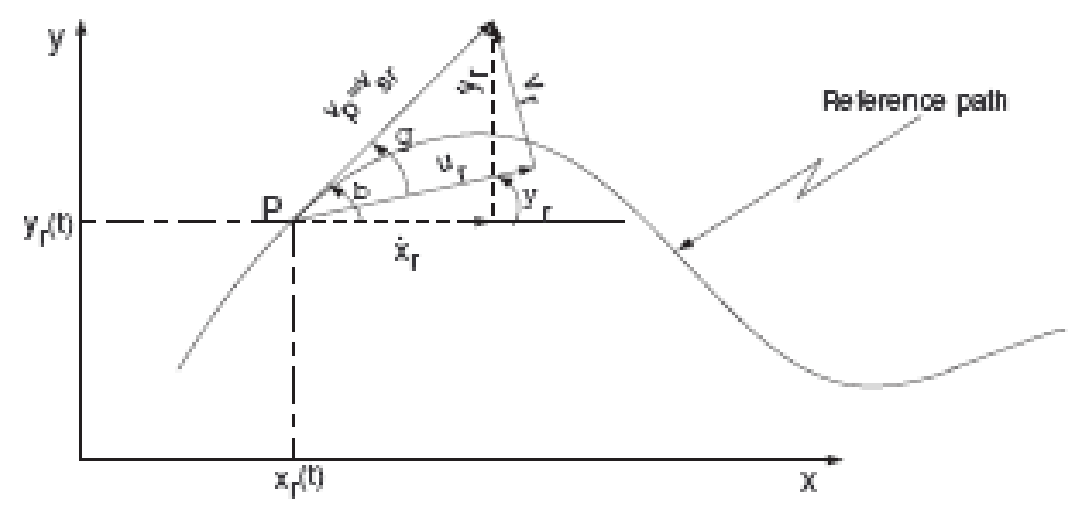

Fig. 2. Ship moving on a planar path.

\section{PATH PLANNING}

In this section, the planning of reference trajectories are analyzed. Since the ship system is underactuated, it is not expected to force the ship to track an arbitrary path. So the only restriction on this trajectory is that it must be sufficiently "smooth" Repoulias and Papadopoulos [2].

\section{Path Kinematics}

Consider Fig. 2, Note that the point $P\left(x_{r}, y_{r}\right)$ is an arbitrary point of the reference trajectory, where $x_{r}$ and $y_{r}$ are the inertial coordinates. So, In order to make the ship follow the reference trajectory we have to make the gravity center coincide with the point $P . v_{p}$ is the magnitude of the velocity vector $v_{p}$ of the reference point $P$ at time $t$. The expression of $v_{p}$ is given by: 


$$
v_{p}=\left\|v_{p}\right\|=\sqrt{\dot{x}_{r}^{2}+\dot{y}_{r}^{2}}
$$

The speed $v_{p}$ is tangential to the reference path on $P$ by making an angle $\beta$ with the inertial axis $(O x)$. Where:

$$
\beta=\arctan \left(\frac{\dot{y}_{r}}{\dot{x}_{r}}\right)
$$

The first and second derivatives of the angle $\beta$ and the velocity $v_{p}$ are given respectively by:

$$
\begin{gathered}
\left\{\begin{array}{l}
\dot{\beta}=\frac{\dot{x}_{r} \ddot{y}_{r}-\dot{y}_{r} \ddot{x}_{r}}{\dot{x}_{r}^{2}+\dot{y}_{r}^{2}} \\
\ddot{\beta}=f_{\beta}\left(\dot{x}_{r}, \ddot{x}_{r}, \dddot{x}_{r}, \dot{y}_{r}, \ddot{y}_{r}, \dddot{y}_{r}\right)
\end{array}\right. \\
\left\{\begin{array}{l}
\dot{v}_{p}=\frac{\dot{x}_{r} \ddot{x}_{r}+\dot{y}_{r} \ddot{y}_{r}}{\sqrt{\dot{x}_{r}^{2}+\dot{y}_{r}^{2}}} \\
\ddot{v}_{p}=f_{v_{p}}\left(\dot{x}_{r}, \ddot{x}_{r}, \dddot{x}_{r}, \dot{y}_{r}, \ddot{y}_{r}, \dddot{y}_{r}\right)
\end{array}\right.
\end{gathered}
$$

\section{Ship's Dynamics on the Path}

After presenting the inertial reference trajectory variables, we will exhibit the algebraic and differential equations that relate the inertial trajectory variables to the local frame velocities, accelerations and orientation. Since the ship tracks the reference trajectory, let assume that $v_{S_{r}}$ is the magnitude of the total linear velocity vector of the gravity $G$ in the ship-fixed frame, which its expression has the following form:

$$
v_{S_{r}}=\left\|v_{S_{r}}\right\|=\sqrt{u_{r}^{2}+v_{r}^{2}}
$$

where $u_{r}$ and $v_{r}$ are the local reference velocity when the ship follows the reference trajectory, see Fig. 2.

The vehicle's total linear velocity $v_{S_{r}}$ is tangential to the reference trajectory, consequently the angle $\psi_{r}$ does not coincide with angle $\beta$, but it differs by angle $\gamma$, where:

$$
\gamma=\arctan \left(\frac{v_{r}}{u_{r}}\right)
$$

Having the description of the reference path and as the ship tracks the path, we can have the two following geometric conditions:

$1^{s t}$ Condition: Equality between the magnitude of the vehicle's total velocity $v_{S_{r}}$ and that of reference path velocity $v_{p}$. As a consequence, we obtain: 


$$
\sqrt{\dot{x}_{r}^{2}+\dot{y}_{r}^{2}}=\sqrt{u_{r}^{2}+v_{r}^{2}}
$$

$2^{\text {nd }}$ Condition: Extraction of the reference angle $\psi_{r}$ from the angles $\beta$ and $\gamma$, observing Fig. 2, we can deduce:

$$
\psi_{r}=\beta-\gamma
$$

Replacing $\beta$ and $\gamma$, respectively, by their expressions in (6) and in (10), yields:

$$
\psi_{r}=\arctan \left(\frac{\dot{y}_{r}}{\dot{x}_{r}}\right)-\arctan \left(\frac{v_{r}}{u_{r}}\right)
$$

Without loss of generality we can assume that the ship moves forward the reference path $\left(u_{r}>0\right)$. Then the sign of the angle $\gamma$ depends on the sign of the reference sway velocity $v_{r}$, which in turn depends on the curvature of the trajectory, i.e. a negative value corresponds to a counterclockwise (CCW) rotation and a positive value to a clockwise (CW) one Repoulias and Papadopoulos [2].

From the equations (5) and (13), $v_{r}$ has the following expression:

$$
v_{r}= \pm \sqrt{\dot{x}_{r}^{2}+\dot{y}_{r}^{2}-u_{r}^{2}}= \pm \sqrt{v_{p}^{2}-u_{r}^{2}}
$$

where \pm indicates the dependence of the sign of $v_{r}$ according to the curvature of the reference trajectory. Differentiating equation (14) yields:

$$
\dot{v}_{r}= \pm \frac{v_{p} \dot{v}_{p}-u_{r} \dot{u}_{r}}{\sqrt{v_{p}^{2}-u_{r}^{2}}}
$$

Remark1: From the equation (14), $u_{r}$ it must verify:

$$
-v_{p} \leq u_{r} \leq v_{p}
$$

where the equality holds in the case of straight line tracking or when a change in the sign of the curvature occurs (then $v_{r}=u_{r}=0$ ).

In order to determine the reference angular velocity and acceleration, differentiating $\psi_{r}$ given in equation (13), respectively, once and twice, yields:

$$
\begin{gathered}
r_{r}=\dot{\psi}_{r}=\dot{\beta} \mp \frac{u_{r} \dot{v}_{p}-\dot{u}_{r} v_{p}}{v_{p} \sqrt{v_{p}^{2}-u_{r}^{2}}} \\
\Rightarrow \dot{r}_{r}=f_{r}\left(\beta, \dot{\beta}, u_{r}, \dot{u}_{r}, \ddot{u}_{r}, v_{p}, \dot{v}_{p}, \ddot{v}_{p}\right)
\end{gathered}
$$

Now, we will focus on computing the surge velocity $u_{r}$. So we distinguish two cases: $1^{\text {st }}$ case (CW-type reference path curvature): Using the fact that $v_{r}>0$, and substituting the appropriate expressions in equation (14),(15) and (17) in the fifth 
equation of the system (4), the following differential equation which solution yields $u_{r}$ is obtained:

$$
\begin{aligned}
& \dot{u}_{r}=\left[\left(m_{11}-m_{22}\right) u_{r} v_{p}^{3}\right]^{-1}\left[-v_{p}^{3}\left(m_{22} v_{p} \dot{v}_{p}+d_{22}\left(v_{p}^{2}-u_{r}^{2}\right)\right)\right. \\
& \left.+m_{11} u_{r}\left(u_{r} \dot{v}_{p} v_{p}^{2}-v_{p} \sqrt{v_{p}^{2}-u_{r}^{2}}\left(\dot{x}_{r} \ddot{y}_{r}-\ddot{x}_{r} \dot{y}_{r}\right)\right)\right]
\end{aligned}
$$

$2^{\text {nd }}$ case (CCW-type reference path curvature): Repeating the same approach used in the first case considering $v_{r}<0$, we obtain:

$$
\dot{u}_{r}=\left[\left(m_{11}-m_{22}\right) u_{r} v_{p}^{3}\right]^{-1}\left[v_{p}^{3}\left(m_{22} v_{p} \dot{v}_{p}+d_{22}\left(v_{p}^{2}-u_{r}^{2}\right)\right)-m_{11} u_{r}\left(u_{r} \dot{v}_{p} v_{p}^{2}+v_{p} \sqrt{v_{p}^{2}-u_{r}^{2}}\left(\dot{x}_{r} \ddot{y}_{r}-\ddot{x}_{r} \dot{y}_{r}\right)\right)\right]
$$

Remark 2: When the curvature of the reference path changes with time, we switch between the above differential equations. What remains now is the computation of the open-loop control efforts. From the forth and the sixth equations of the system (4) the controls' expressions are given by:

$$
\left\{\begin{array}{l}
\tau_{u r}=m_{11} \dot{u}_{r}+d_{11} u_{r}-m_{22} v_{r} r_{r} \\
\tau_{r r}=m_{33} \dot{r}_{r}-\frac{\left(m_{11}-m_{22}\right)}{m_{33}} r_{r} u_{r}+d_{33} r_{r}
\end{array}\right.
$$

In the next section, we will focus on concepting control rules of auto-pilot ship which are derived for nonlinear controllers designed with the aid of the Backstepping method Krstić et. al. [9].

\section{CONTROL DESIGN}

This section is divided in two subsections. In the first one we will write the error dynamic model, and in the second subsection we will use the Backstepping method Krstić et. al. [9] that provides a non linear control law that relies on the tracking control objective.

This Backstepping technique overcome the difficulty of Lyapunov function construction, it allows us to build iteratively the adapted Lyapunov function to the system and allows us to deduct the command(order) which returns the derivative of the Lyapunov function defined negative.

\section{Error System}

In this section, configuration error and velocity error will define as:

$$
\left\{\begin{array} { l } 
{ x _ { e } = x - x _ { r } } \\
{ y _ { e } = y - y _ { r } } \\
{ \psi _ { e } = \psi - \psi _ { r } }
\end{array} \quad \left\{\begin{array}{l}
u_{e}=u-u_{r} \\
v_{e}=v-v_{r} \\
r_{e}=r-r_{r}
\end{array}\right.\right.
$$

The error kinematics is obtained by differentiating the system that present the configuration error in (21): 


$$
\left\{\begin{array}{l}
\dot{X}_{e}=R U_{e}+\lambda \\
\dot{\psi}_{e}=r_{e}
\end{array}\right.
$$

where $R$ is a square matrix, that has the following form:

$$
R=\left[\begin{array}{cc}
c_{\psi} & -s_{\psi} \\
s_{\psi} & c_{\psi}
\end{array}\right]
$$

$U_{e}=\left[u_{e}, v_{e}\right]^{T}$ is the linear velocity error vector, $X_{e}=\left[x_{e}, y_{e}\right]^{T}$ is the position error vector and $\lambda=R_{\psi, \psi_{r}} U_{r}$ where

$$
R_{\psi, \psi_{r}}=\left[\begin{array}{cc}
c_{\psi}-c_{\psi_{r}} & -s_{\psi}+s_{\psi_{r}} \\
s_{\psi}-s_{\psi_{r}} & c_{\psi}-c_{\psi_{r}}
\end{array}\right]
$$

and $U_{r}=\left[u_{r}, v_{r}\right]^{T}$ is the reference linear velocity vector.

Differentiating the second system of (21), we obtain the error dynamics:

$$
\left\{\begin{array}{l}
\dot{u}_{e}=\tau_{1} \\
\dot{v}_{e}=-\frac{m_{11}}{m_{22}}\left(u_{e} r_{e}+u_{e} r_{r}+u_{r} r_{e}\right)-\frac{d_{22}}{m_{22}} v_{e}-\dot{v}_{r}-\frac{m_{11}}{m_{22}} u_{r} r_{r}-\frac{d_{22}}{m_{22}} v_{r} \\
\dot{r}_{e}=\tau_{3}
\end{array}\right.
$$

where the expressions of $\tau_{1}$ and $\tau_{3}$ are:

$$
\left\{\begin{array}{l}
\tau_{1}=\frac{m_{22}}{m_{11}}\left(v_{e} r_{e}+v_{e} r_{r}+v_{r} r_{e}\right)-\frac{d_{11}}{m_{11}} u_{e}-\dot{u}_{r}+\frac{m_{22}}{m_{11}} v_{r} r_{r}-\frac{d_{11}}{m_{11}} v_{r}+\frac{1}{m_{11}} \tau_{u} \\
\tau_{3}=\frac{\left(m_{11}-m_{22}\right)}{m_{33}}\left(v_{e} u_{e}+v_{e} u_{r}+v_{r} u_{e}\right)-\frac{d_{33}}{m_{33}} r_{e}-\dot{r}_{r}+\frac{\left(m_{11}-m_{22}\right)}{m_{33}} v_{r} u_{r}-\frac{d_{33}}{m_{33}} r_{r}+\frac{1}{m_{33}} \tau_{r}
\end{array}\right.
$$

According to systems (22) and (25), the complete error model of the ship is:

$$
\left\{\begin{array}{l}
\dot{X}_{e}=R U_{e}+\lambda \\
\dot{\psi}_{e}=r_{e} \\
\dot{u}_{e}=\tau_{1} \\
\dot{v}_{e}=-\frac{m_{11}}{m_{22}}\left(u_{e} r_{e}+u_{e} r_{r}+u_{r} r_{e}\right)-\frac{d_{22}}{m_{22}} v_{e}-\dot{v}_{r}-\frac{m_{11}}{m_{22}} u_{r} r_{r}-\frac{d_{22}}{m_{22}} v_{r} \\
\dot{r}_{e}=\tau_{3}
\end{array}\right.
$$

Consequently, the control problem is then transformed into a stabilization problem of the error dynamics (27). 


\section{Error System Stabilization}

The error system define in the following section is now used to design the backstepping algorithm which guaranteed the reference trajectory tracking objective. The control design is described step by step as follows:

step 1: In the first step, we focus on stabilizing the state variables $\left(x_{e}, y_{e}\right)$. Introducing the following Lyapunov function candidate:

$$
V_{1}=\frac{1}{2} X_{e}^{T} X_{e}
$$

then, the time derivative is expressed as:

$$
\dot{V}_{1}=X_{e}^{T}\left(R U_{e}+\lambda\right)
$$

We can treat $U_{e}$ as an auxiliary control. We will choose a virtual control $\left[\alpha_{u}, \alpha_{v}\right]^{T}$ instead of $U_{e}$ as:

$$
\left[\alpha_{u}, \alpha_{v}\right]^{T}=-R^{T}\left[K+K_{1}\right] X_{e}
$$

with $K$ and $K_{1}$ are positive definite square matrices. Substituting (29) into (30), we get:

$$
\dot{V}_{1}=X_{e}^{T}\left[-\left(K+K_{1}\right) X_{e}+\lambda\right]=-X_{e}^{T} K X_{e}-k_{1}\left(x_{e}-\frac{\lambda_{1}}{2 k_{1}}\right)^{2}-k_{1}\left(y_{e}-\frac{\lambda_{2}}{2 k_{1}}\right)^{2}+\frac{\|\lambda\|^{2}}{4 k_{1}}
$$

where $\lambda=\left[\lambda_{1}, \lambda_{2}\right]^{T}$ (The quantity $\lambda$ is a bounded function). When we increase the gain $K_{1}$ the quantity $\frac{\|\lambda\|^{2}}{4 k_{1}}$ diminishes.

step 2: The components of the vector $U_{e}$ are not a true control, we need to introduce new error variables $z_{u}$ and $z_{v}$ that we would derive to zero

$$
\left\{\begin{array}{l}
z_{u}=u_{e}-\alpha_{u} \\
z_{v}=v_{e}-\alpha_{v}
\end{array}\right.
$$

Then, (31) gives:

$$
\dot{V}_{1}=X_{e}^{T}\left[-\left(K+K_{1}\right) X_{e}+R Z_{u}+\lambda\right]
$$

where $Z_{u}=\left[z_{u}, z_{v}\right]^{T}$.

The task now is to derive $z_{u}$ to zero, so we consider the following Lyapunov function candidate 


$$
V_{2}=V_{1}+\frac{1}{2} z_{u}^{2}
$$

Using (33) and (34), the derivative of $V_{2}$ can be derived as follows:

$$
\begin{aligned}
& \dot{V}_{2}=-X_{e}^{T} K X_{e}-k_{1}\left(x_{e}-\frac{\lambda_{1}}{2 k_{1}}\right)^{2}-k_{1}\left(y_{e}-\frac{\lambda_{2}}{2 k_{1}}\right)^{2}+\frac{\|\lambda\|^{2}}{4 k_{1}}+z_{v}\left(y_{e} c_{\psi}-x_{e} s_{\psi}\right) \\
& +z_{u}\left[\tau_{1}+\left(k+k_{1}\right) u_{e}+\left(k+k_{1}\right)\left(\lambda_{1} c_{\psi}+\lambda_{2} s_{\psi}\right)+x_{e} c_{\psi}+y_{e} s_{\psi}\right]
\end{aligned}
$$

According to (35), the control law $\tau_{1}$ is designed as follows:

$$
\tau_{1}=-c_{1} z_{u}-\left(k+k_{1}\right) u_{e}-\left(k+k_{1}\right)\left(\lambda_{1} c_{\psi}+\lambda_{2} s_{\psi}\right)-x_{e} c_{\psi}-y_{e} s_{\psi}+f_{u}
$$

where $c_{1}$ is a positive constant and $f_{u}$ is a design variable for subsequent use. Substituting equation (36) into (35) the following equation can be obtained:

$$
\dot{V}_{2}=-X_{e}^{T} K X_{e}-k_{1}\left(x_{e}-\frac{\lambda_{1}}{2 k_{1}}\right)^{2}-k_{1}\left(y_{e}-\frac{\lambda_{2}}{2 k_{1}}\right)^{2}+\frac{\|\lambda\|^{2}}{4 k_{1}}+z_{v}\left(y_{e} c_{\psi}-x_{e} s_{\psi}\right)-c_{1} z_{u}^{2}+z_{u} f_{u}
$$

So far, the controlled subsystem of the linear kinematics and the error $z_{u}$ is transformed to:

$$
\left\{\begin{array}{l}
\dot{X}_{e}=-\left(K+K_{1}\right) X_{e}+R Z_{u}+\lambda \\
\dot{z}_{u}=-c_{1} z_{u}-x_{e} c_{\psi}-y_{e} s_{\psi}+f_{u}
\end{array}\right.
$$

step 3: In this step we consider the stabilization of the subsystem that is controlled by the assumed auxiliary control $r_{e}$ (the rotational kinematics and the sway dynamic error $z_{v}$ ). Before starting, we make some manipulations on $z_{v}$, in order to show the virtual control $r_{e}$ and $z_{u}$. The dynamic $z_{v}$ is written as:

$$
\begin{aligned}
& \dot{z}_{v}=-\frac{m_{11}}{m_{22}} u r_{e}-\frac{m_{11}}{m_{22}} z_{u} r_{r}-\frac{m_{11}}{m_{22}} \alpha_{u} r_{r}-\frac{d_{22}}{m_{22}} v_{e}-\dot{v}_{r}-\frac{m_{11}}{m_{22}} u_{r} r_{r}-\frac{d_{22}}{m_{22}} v_{r}+\left(k+k_{1}\right) v_{e} \\
& -\left(k+k_{1}\right)\left(\lambda_{1} s_{\psi}-\lambda_{2} c_{\psi}\right)
\end{aligned}
$$

which gives:

$$
\dot{z}_{v}=-\frac{m_{11}}{m_{22}} u r_{e}-\frac{m_{11}}{m_{22}} z_{u} r_{r}+\delta_{v}
$$

where

$$
\delta_{v}=-\frac{m_{11}}{m_{22}} \alpha_{u} r_{r}-\frac{d_{22}}{m_{22}} v_{e}-\dot{v}_{r}-\frac{m_{11}}{m_{22}} u_{r} r_{r}-\frac{d_{22}}{m_{22}} v_{r}+\left(k+k_{1}\right) v_{e}-\left(k+k_{1}\right)\left(\lambda_{1} s_{\psi}-\lambda_{2} c_{\psi}\right)
$$

Assuming $u \neq 0$ is a natural condition for tracking, it means, if $u=0$ we can not act on our system with $r_{e}$ as a control. We note a virtual control law $\alpha_{r}$ for $r_{e}$. To design $\alpha_{r}$, add terms concerning $\psi_{e}$ and $z_{v}$ to $V_{2}$ to form the following Lyapunov Function: 


$$
V_{3}=V_{2}+\frac{1}{2}\left(z_{u}^{2}+\psi_{e}^{2}\right)
$$

The time derivative of $V_{3}$ is:

$$
\begin{aligned}
& \dot{V}_{3}=-X_{e}^{T} K X_{e}-k_{1}\left(x_{e}-\frac{\lambda_{1}}{2 k_{1}}\right)^{2}-k_{1}\left(y_{e}-\frac{\lambda_{2}}{2 k_{1}}\right)^{2}+\frac{\|\lambda\|^{2}}{4 k_{1}}+z_{v}\left(y_{e} c_{\psi}-x_{e} s_{\psi}+\delta_{v}\right)-c_{1} z_{u}^{2} \\
& +z_{u}\left(f_{u}-\frac{m_{11}}{m_{22}} z_{v} r_{r}\right)+\alpha_{r}\left(\psi_{e}-\frac{m_{11}}{m_{22}} u z_{v}\right)
\end{aligned}
$$

A simpler virtual control law $\alpha_{r}$ is chosen:

$$
\alpha_{r}=-c_{r}\left(\psi_{e}-\frac{m_{11}}{m_{22}} u z_{v}\right)
$$

where $c_{r}>0$ is a design constant. Taking into account (44), the time derivative of $V_{3}$ becomes:

$$
\begin{aligned}
& \dot{V}_{3}=-X_{e}^{T} K X_{e}-k_{1}\left(x_{e}-\frac{\lambda_{1}}{2 k_{1}}\right)^{2}-k_{1}\left(y_{e}-\frac{\lambda_{2}}{2 k_{1}}\right)^{2}+\frac{\|\lambda\|^{2}}{4 k_{1}}+z_{v}\left(y_{e} c_{\psi}-x_{e} s_{\psi}+\delta_{v}\right)-c_{1} z_{u}^{2} \\
& +z_{u}\left(f_{u}-\frac{m_{11}}{m_{22}} z_{v} r_{r}\right)-c_{r}\left(\psi_{e}-\frac{m_{11}}{m_{22}} u z_{v}\right)^{2}
\end{aligned}
$$

step 4: As we know that $r_{e}$ is not a true control, we will introduce the final error variable $z_{r}=r_{e}-\alpha_{r}$ instead of $\alpha_{r}$ and we use $\tau_{3}$ to stabilize the subsystem.

$$
\left\{\begin{array}{l}
\dot{z}_{v}=-\frac{m_{11}}{m_{22}} u\left(z_{r}+\alpha_{r}\right)-\frac{m_{11}}{m_{22}} z_{u} r_{r}+\delta_{v} \\
\dot{\psi}_{e}=z_{r}+\alpha_{r} \\
\dot{z}_{r}=\tau_{3}-\dot{\alpha}_{r}
\end{array}\right.
$$

With

$$
\dot{\alpha}_{r}=-c_{r}\left[1+\left(\frac{m_{11}}{m_{22}} u\right)^{2}\right]\left(z_{r}+\alpha_{r}\right)+c_{r} \frac{m_{11}}{m_{22}} u \delta_{v}-c_{r}\left(\frac{m_{11}}{m_{22}}\right)^{2} u z_{u} r_{r}
$$

Then, after the set of transformation, the controlled dynamic system so far is: 


$$
\left\{\begin{array}{l}
\dot{X}_{e}=-\left(K+K_{1}\right) X_{e}+R Z_{u}+\lambda \\
\dot{z}_{u}=-c_{1} z_{u}-x_{e} c_{\psi}-y_{e} s_{\psi}+f_{u} \\
\dot{z}_{v}=-\frac{m_{11}}{m_{22}} u z_{r}+c_{r} \frac{m_{11}}{m_{22}} u \psi_{e}-c_{r}\left(\frac{m_{11}}{m_{22}} u\right)^{2} z_{v}-\frac{m_{11}}{m_{22}} z_{u} r_{r}+\delta_{v} \\
\dot{\psi}_{e}=z_{r}-c_{r} \psi_{e}+c_{r} \frac{m_{11}}{m_{22}} u z_{v} \\
\dot{z}_{r}=\tau_{3}-\dot{\alpha}_{r}
\end{array}\right.
$$

Again, to stabilize the error variable $z_{r}$, we consider the following Lyapunov function candidate:

$$
V_{4}=V_{3}+\frac{1}{2} z_{r}^{2}
$$

and computing its time derivative, we obtain:

$$
\begin{aligned}
& \dot{V}_{4}=-X_{e}^{T} K X_{e}-k_{1}\left(x_{e}-\frac{\lambda_{1}}{2 k_{1}}\right)^{2}-k_{1}\left(y_{e}-\frac{\lambda_{2}}{2 k_{1}}\right)^{2}+\frac{\|\lambda\|^{2}}{4 k_{1}}+z_{v}\left(y_{e} c_{\psi}-x_{e} s_{\psi}+\delta_{v}\right)-c_{1} z_{u}^{2} \\
& +z_{u}\left(f_{u}-\frac{m_{11}}{m_{22}} z_{v} r_{r}\right)-c_{r}\left(\psi_{e}-\frac{m_{11}}{m_{22}} u z_{v}\right)^{2}+z_{r}\left[\tau_{3}+c_{r}\left[1+\left(\frac{m_{11}}{m_{22}} u\right)^{2}\right]\left(z_{r}-c_{r} \psi_{e}+c_{r} \frac{m_{11}}{m_{22}} u z_{v}\right)\right. \\
& \left.-c_{r} \frac{m_{11}}{m_{22}} u \delta_{v}+c_{r}\left(\frac{m_{11}}{m_{22}}\right)^{2} u z_{u} r_{r}+\psi_{e}-\frac{m_{11}}{m_{22}} u z_{v}\right]
\end{aligned}
$$

Choosing

$$
\tau_{3}=-c_{r}\left[1+\left(\frac{m_{11}}{m_{22}} u\right)^{2}\right] r_{e}+c_{r} \frac{m_{11}}{m_{22}} u \delta_{v}-c_{r}\left(\frac{m_{11}}{m_{22}}\right)^{2} u z_{u} r_{r}-\psi_{e}+\frac{m_{11}}{m_{22}} u z_{v}-c z_{r}
$$

and

$$
f_{u}=\frac{m_{11}}{m_{22}} z_{v} r_{r}-c_{4} z_{u} z_{v}^{2}
$$

with $c$ and $c_{4}$ are positive constants. Substituting equations (51) and (52) in (50) we obtain:

$$
\begin{aligned}
& \dot{V}_{4}=-X_{e}^{T} K X_{e}-k_{1}\left(x_{e}-\frac{\lambda_{1}}{2 k_{1}}\right)^{2}-k_{1}\left(y_{e}-\frac{\lambda_{2}}{2 k_{1}}\right)^{2}+\frac{\|\lambda\|^{2}}{4 k_{1}}+z_{v}\left(y_{e} c_{\psi}-x_{e} s_{\psi}+\delta_{v}\right) \\
& -c_{1} z_{u}^{2}-c z_{r}^{2}-c_{r} \psi_{e}^{2}-c_{4} z_{u}^{2} z_{v}^{2}+2 c_{r} \frac{m_{11}}{m_{22}} u z_{v} \psi_{e}-c_{r}\left(\frac{m_{11}}{m_{22}} u z_{v}\right)^{2}
\end{aligned}
$$

In the above expression we remark that the last three terms have uncertain signs, so we will examine them by considering all of them are positive. For the analysis we will 
use the inequality of Young (Ghommam [10]), with the quantities $\varepsilon_{i}, \quad i=1 \ldots .6$ are positive constants.

$$
\begin{gathered}
z_{v}\left(y_{e} c_{\psi}-x_{e} s_{\psi}\right) \leq \frac{1}{4 \varepsilon_{1}}\left|y_{e}\right|^{2}+\varepsilon_{1}\left|z_{v}\right|^{2}+\frac{1}{4 \varepsilon_{1}}\left|x_{e}\right|^{2}+\varepsilon_{1}\left|z_{v}\right|^{2} \\
2 c_{r}\left(\frac{m_{11}}{m_{22}} u\right) z_{v} \psi_{e} \leq \frac{1}{\varepsilon_{2}}\left(\frac{m_{11}}{m_{22}} u\right)^{2}\left|\psi_{e}\right|^{2}+\varepsilon_{2} c_{r}{ }^{2}\left|z_{v}\right|^{2}
\end{gathered}
$$

Now, we will expand the expression of $z_{v} \delta_{v}$, we obtain the following expression:

$$
\begin{aligned}
& z_{v} \delta_{v}=\frac{m_{11}}{m_{22}} r_{r}\left(k+k_{1}\right)\left(x_{e} c_{\psi}-y_{e} s_{\psi}\right) z_{v}+\left(\left(k+k_{1}\right)-\frac{d_{22}}{m_{22}}\right) v_{e} z_{v}-\left(\dot{v}_{r}+\frac{m_{11}}{m_{22}} u_{r} r_{r}+\frac{d_{22}}{m_{22}} v_{r}\right) z_{v} \\
& -\left(k+k_{1}\right)\left(v_{r} c_{\psi_{e}}-u_{r} s_{\psi_{e}}-v_{r}\right) z_{v}
\end{aligned}
$$

Then, we will bound the four terms of (56) one by one as follows:

$$
\begin{gathered}
\frac{m_{11}}{m_{22}} r_{r}\left(k+k_{1}\right)\left(x_{e} c_{\psi}-y_{e} s_{\psi}\right) z_{v} \leq \frac{1}{4 \varepsilon_{3}}\left(k+k_{1}\right)^{2}\left|r_{r}\right|^{2}\left|x_{e}\right|^{2}+\varepsilon_{3}\left(\frac{m_{11}}{m_{22}}\right)^{2}\left|z_{v}\right|^{2} \\
+\frac{1}{4 \varepsilon_{3}}\left(k+k_{1}\right)^{2}\left|r_{r}\right|^{2}\left|y_{e}\right|^{2}+\varepsilon_{3}\left(\frac{m_{11}}{m_{22}}\right)^{2}\left|z_{v}\right|^{2} \\
\left(\left(k+k_{1}\right)-\frac{d_{22}}{m_{22}}\right) v_{e} z_{v} \leq \frac{1}{4 \varepsilon_{4}}\left(\left(k+k_{1}\right)-\frac{d_{22}}{m_{22}}\right)^{2}\left|v_{e}\right|^{2}+\varepsilon_{4}\left|z_{v}\right|^{2} \\
-\left(\dot{v}_{r}+\frac{m_{11}}{m_{22}} u_{r} r_{r}+\frac{d_{22}}{m_{22}} v_{r}\right) z_{v} \leq\left.\varepsilon_{5}\left(\dot{v}_{r}+\frac{m_{11}}{m_{22}} u_{r} r_{r}+\frac{d_{22}}{m_{22}} v_{r}\right)\right|^{2}+\frac{1}{4 \varepsilon_{5}}\left|z_{v}\right|^{2} \\
-\left(k+k_{1}\right)\left(v_{r} c_{\psi_{e}}-u_{r} s_{\psi_{e}}-v_{r}\right) z_{v} \leq\left.\varepsilon_{6}\left(k+k_{1}\right)^{2}\left|\left(v_{r} c_{\psi_{e}}-u_{r} s_{\psi_{e}}-v_{r}\right)^{2}+\frac{1}{4 \varepsilon_{6}}\right| z_{v}\right|^{2}
\end{gathered}
$$

Taking into account the results from (54) to (60), the derivative of $V_{4}$ in (53), will be increased by:

$$
\begin{aligned}
& \dot{V}_{4} \leq-\left[k-\frac{1}{4 \varepsilon_{1}}-\frac{1}{4 \varepsilon_{3}}\left(k+k_{1}\right)^{2} r_{\max }^{2}\right] x_{e}^{2}-\left[k-\frac{1}{4 \varepsilon_{1}}-\frac{1}{4 \varepsilon_{3}}\left(k+k_{1}\right)^{2} r_{\max }^{2}\right] y_{e}^{2}-k_{1}\left(x_{e}-\frac{\lambda_{1}}{2 k_{1}}\right)^{2} \\
& -k_{1}\left(y_{e}-\frac{\lambda_{2}}{2 k_{1}}\right)^{2}+\frac{\left\|\lambda_{\max }\right\|^{2}}{4 k_{1}}-c_{1} z_{u}^{2}-c_{r}\left(\frac{m_{11}}{m_{22}} u_{\max } z_{v}\right)^{2}-\left(c_{r}-\frac{1}{\varepsilon_{2}}\left(\frac{m_{11}}{m_{22}} u_{\max }\right)^{2}\right) \psi_{e}^{2}-c_{r} z_{r}^{2} \\
& -\left(c_{4} z_{u}^{2}-2 \varepsilon_{1}-\varepsilon_{2} c_{r}^{2}-2 \varepsilon_{3}\left(\frac{m_{11}}{m_{22}}\right)^{2}-\varepsilon_{4}-\frac{1}{4 \varepsilon_{5}}-\frac{1}{4 \varepsilon_{6}}\right) z_{v}^{2}+\frac{1}{4 \varepsilon_{4}}\left[\left(k+k_{1}\right)-\frac{d_{22}}{m_{22}}\right]^{2} v_{e \max }^{2}+\varepsilon_{5}\left|\zeta_{1}\right|^{2} \\
& +\varepsilon_{6}\left(k+k_{1}\right)^{2}\left|\zeta_{2}\right|^{2}
\end{aligned}
$$


where $r_{\max }, \lambda_{\max }, v_{\max }, u_{r_{\max }}$ and $v_{e \max }$ are the maximum values for the time varying quantities. In the inequality $(61)$ the term $c_{4} z_{u}^{2}-2 \varepsilon_{1}-\varepsilon_{2} c_{r}^{2}-2 \varepsilon_{3}\left(\frac{m_{11}}{m_{22}}\right)^{2}-\varepsilon_{4}-\frac{1}{4 \varepsilon_{5}}-\frac{1}{4 \varepsilon_{6}}$ must be positive, for that we set $2 \varepsilon_{1}+\varepsilon_{2} c_{r}^{2}+2 \varepsilon_{3}\left(\frac{m_{11}}{m_{22}}\right)^{2}+\varepsilon_{4}+\frac{1}{4 \varepsilon_{5}}+\frac{1}{4 \varepsilon_{6}}$. So, it must that $c_{4} z_{u}^{2}>\zeta \Rightarrow z_{u}>\sqrt{\frac{\zeta}{c_{4}}}$ which is verified for a large and positive $c_{4}$ and small $\zeta$ consequently we have:

$$
\begin{aligned}
& \dot{V}_{4} \leq-\varpi_{1} x_{e}^{2}-\varpi_{1} y_{e}^{2}-k_{1}\left(x_{e}-\frac{\lambda_{1}}{2 k_{1}}\right)^{2}-k_{1}\left(y_{e}-\frac{\lambda_{2}}{2 k_{1}}\right)^{2}-c_{1} z_{u}^{2}-\varpi_{3} \psi_{e}^{2}-c_{r} z_{r}^{2}-\left[c_{4} z_{u}^{2}-\zeta\right] z_{v}^{2} \\
& -\varpi_{2} z_{v}^{2}+\mu
\end{aligned}
$$

where

$$
\begin{aligned}
& \varpi_{1}=\left[k-\frac{1}{4 \varepsilon_{1}}-\frac{1}{4 \varepsilon_{3}}\left(k+k_{1}\right)^{2} r_{\max }^{2}\right] \\
& \varpi_{2}=c_{r}\left(\frac{m_{11}}{m_{22}} u_{\max }\right)^{2} \\
& \varpi_{3}=c_{r}-\frac{1}{\varepsilon_{2}}\left(\frac{m_{11}}{m_{22}} u_{\max }\right)^{2} \\
& \mu=\frac{1}{4 \varepsilon_{4}}\left[\left(k+k_{1}\right)-\frac{d_{22}}{m_{22}}\right]^{2} v_{e \text { max }}^{2}+\varepsilon_{5}\left|\zeta_{1}\right|^{2}+\varepsilon_{6}\left(k+k_{1}\right)^{2}\left|\zeta_{2}\right|^{2}+\frac{\left\|\lambda_{\max }\right\|^{2}}{4 k_{1}}
\end{aligned}
$$

which are all positive. So, from the inequality (62), we can deduce that:

$$
\begin{aligned}
& \dot{V}_{4} \leq-\varpi_{1} x_{e}^{2}-\varpi_{1} y_{e}^{2}-c_{1} z_{u}^{2}-\varpi_{2} z_{v}^{2}-\varpi_{3} \psi_{e}^{2}-c_{r} z_{r}^{2}+\mu \\
& \leq v V_{4}+\mu
\end{aligned}
$$

where $v=\min \left\{\varpi_{1}, c_{1}, \varpi_{2}, \varpi_{3}, c_{4}\right\}$.

By employing the comparison lemma (Khallil [11]), we can yield:

$$
V_{4}(t) \leq V_{4}(0) e^{-2 v t}+\frac{\mu}{2 v}, t \geq 0
$$

Which implies that the states of error $\left(x_{e}, y_{e}, \psi_{e}, z_{u}, z_{v}, z_{r}\right)$ remain in a bounded set about the origin. 


\section{ANALATYCAL RESULTS}

In this section, we carry out some computer simulations that illustrate the performance that can be achieved with the motion control algorithms mentioned before. The simulations are implemented on Mat lab. We assume the underactuated ship vehicle model with the following parameters of kinematics and the dynamics that are the same as those in Dong and Guo [12]:

$m_{11}=200 \mathrm{Kg}, m_{22}=250 \mathrm{Kg}, m_{33}=80 \mathrm{Kg}, d_{11}=70 \mathrm{Kg} / \mathrm{s}, d_{22}=100 \mathrm{Kg} / \mathrm{s}$ and $d_{33}=50 \mathrm{Kg} / \mathrm{s}$

Here, we will track a sinusoidal path (time varying trajectory). Responses were obtained for two cases: in the first case we will show simulation results of the tracking without disturbances and in the second case we will present simulation results in the disturbed condition, in this case we assume that the disturbances are the sea drag forces which are taken equal to $0.05 u$ and $0.05 v$ acting in the surge and sway respectively. We choose a desired trajectory which its parametric system describing by:

$$
\left\{\begin{array}{l}
x_{r}=0.03 t \\
y_{r}=10 \sin (0.03 t)
\end{array}\right.
$$

For simulation uses, we pick the following initial condition for the real system:

$$
[x(0), y(0), \psi(0), u(0), v(0), r(0)]=[0.12,2.5,0,0,0,0]
$$

and the initial desired conditions are:

$$
\left[x_{r}(0), y_{r}(0), \psi_{r}(0), u_{r}(0), v_{r}(0), r_{r}(0)\right]=[0,0,1.3175,0.3,0.03,0.2]
$$

The control parameters used in the Backstepping control are set to $k_{1}=0.01, k=0.03, c_{1}=10, c_{r}=3, c=10$ and $c_{4}=1$ to satisfy convergence conditions.

\section{Sinusoidal Tracking with No Disturbances}

Figure 3 shows the desired trajectory and the actual trajectory of the ship in $X-Y$ plane. We see that the ship converged to the desired trajectory. For each figure of Figs. (4-7), we have figures on the left side and on the right side. The figures on the left regard simulation time of 450 s needed for two full period for the sinus function, while the figures on the right regard the first $70 \mathrm{~s}$ in order to observe the transient responses.

The simulations results, depicted in Figs. (4-5), are for response of positions $(x, y)$ and linear velocities $(u, v)$, we observe that after a transition period of $65 \mathrm{~s}$ all presented variables converge to the desired variables. Figure 6 shows the convergence of $\psi$ and yaw velocity $r$ to $\psi_{r}$ and $r_{r}$, respectively after around $2 s$. In Figure 7 , we see the behavior of the control surge force $\tau_{u}$ and the control yaw torque $\tau_{r}$ needed for tracking and they also converge to the open-loop control efforts $\tau_{u r}$ and $\tau_{r r}$ respectively after around $2 s$. 


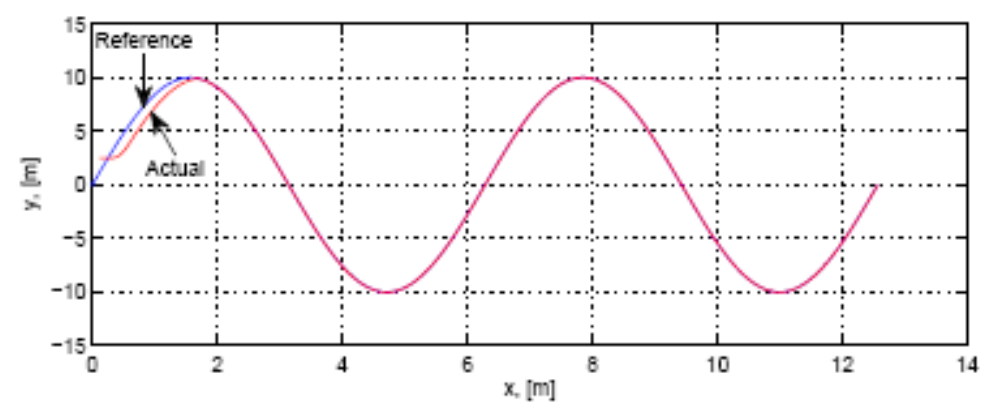

Fig. 3. Ship reference and actual path.
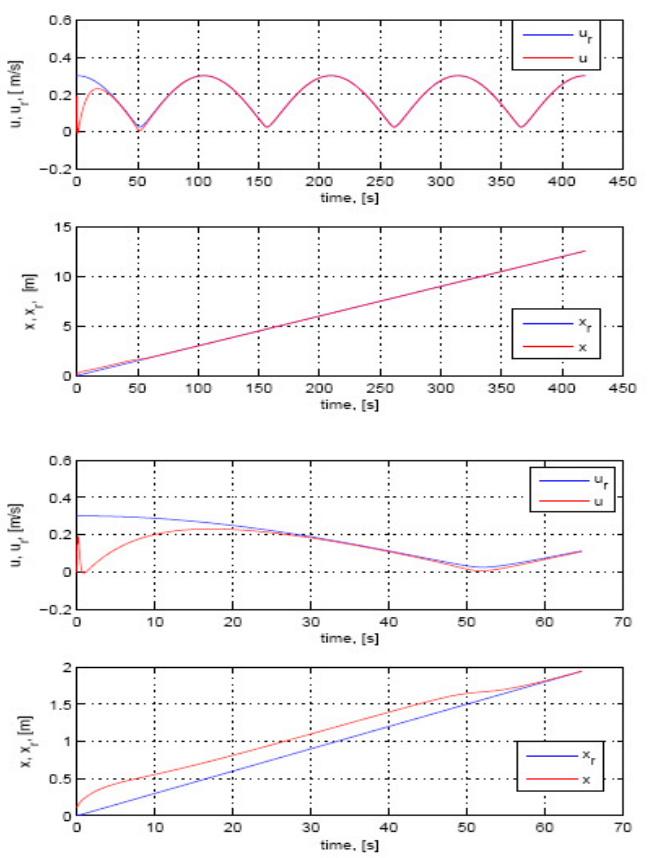

Fig. 5. (left side) Time responses of $y$ and $v$ together with $y_{r}$ and $v_{r}$
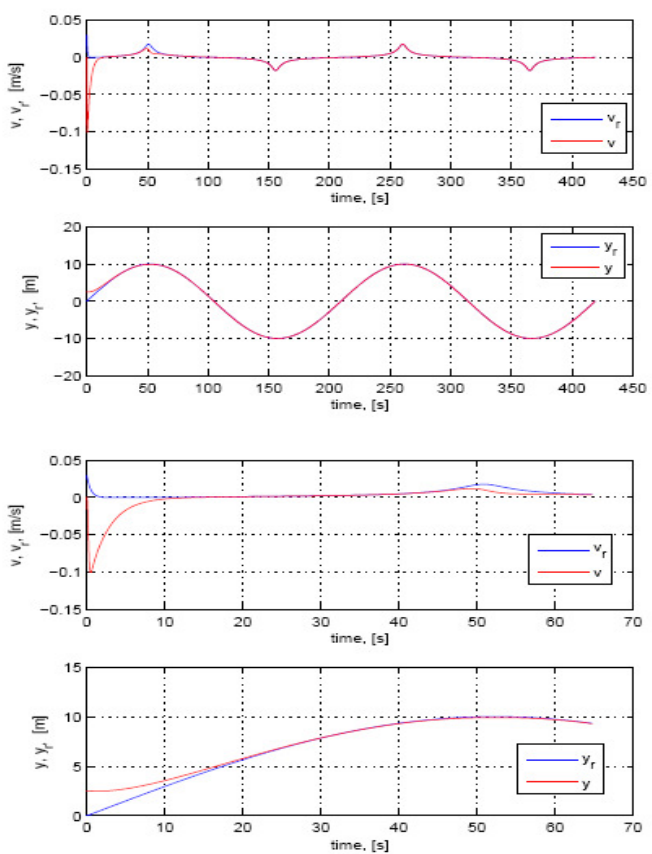

Fig. 4. ( right side) Time responses of $x$ and $u$ together with $x_{r}$ and $u_{r}$

\section{Sinusoidal Tracking Path with Disturbances}

In the disturbed condition, we present some numerical results concerning the robustness of the designed control law. Figs. (8-12) are simulation results with disturbance in which we assume that there are added drag forces of the $\operatorname{order}(0.05 u, 0.05 v)$. As in the preceding section, we present in each figure the response after two full periods on the left side and on the right the first $65 \mathrm{~s}$. We remark that the proposed control law is robust for the chosen disturbances. 

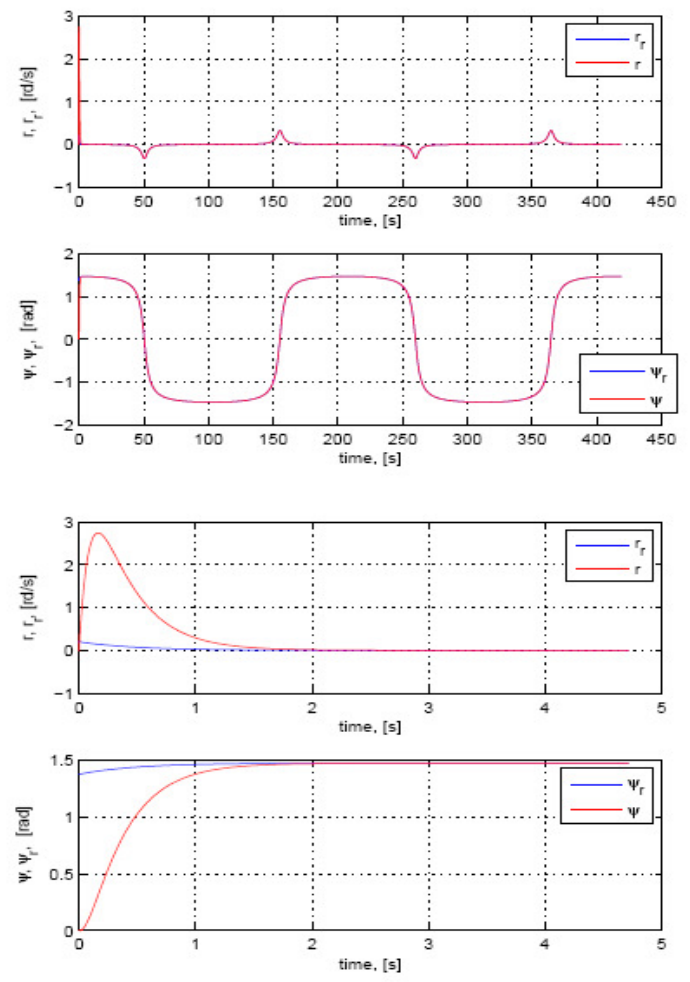

Fig. 7. (left side) Time responses of $\tau_{u}$ and $\tau_{r}$ together with $\tau_{u_{r}}$ and $\tau_{r_{r}}$
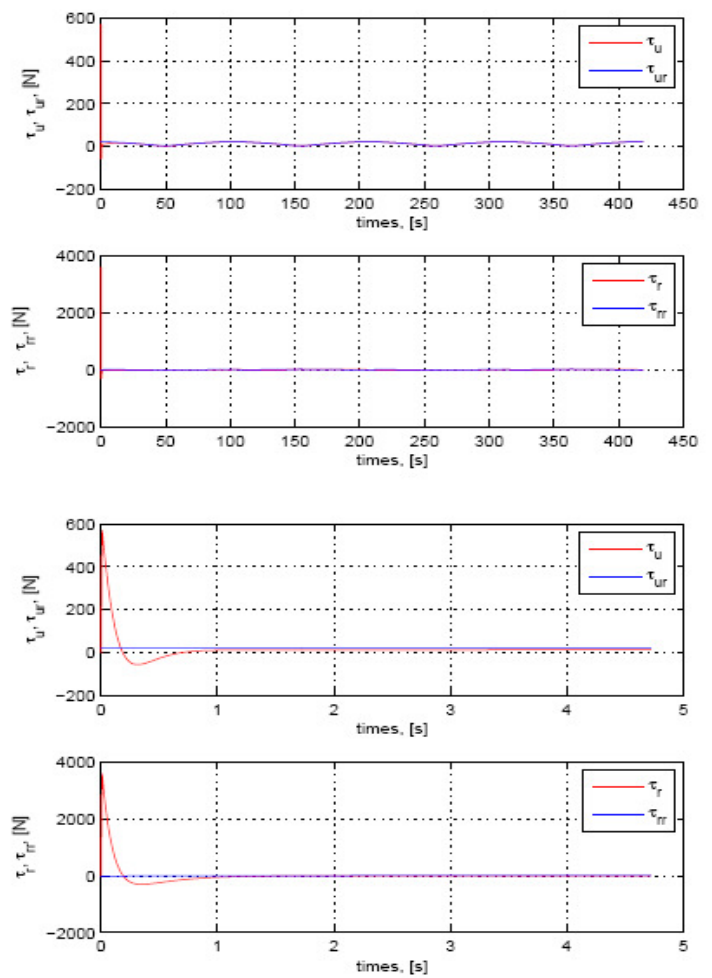

Fig. 6. (right side) Time responses of $\psi$ and $r$ together with $\psi_{r}$ and $r_{r}$

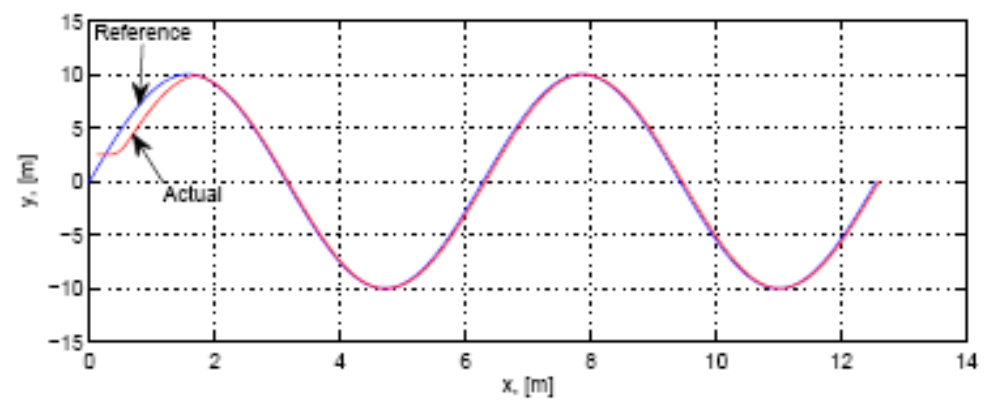

Fig. 8. The trajectory of the ship in the $X-Y$ plane and the desired trajectory. 

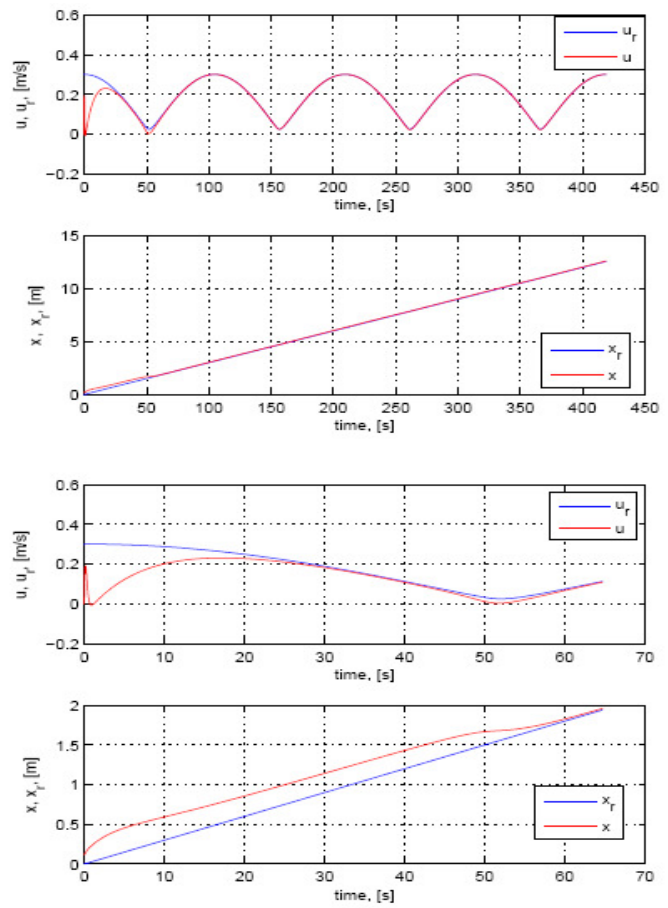

Fig. 10. (left side)Time responses of $y$ and $v$ together with $y_{r}$ and $v_{r}$
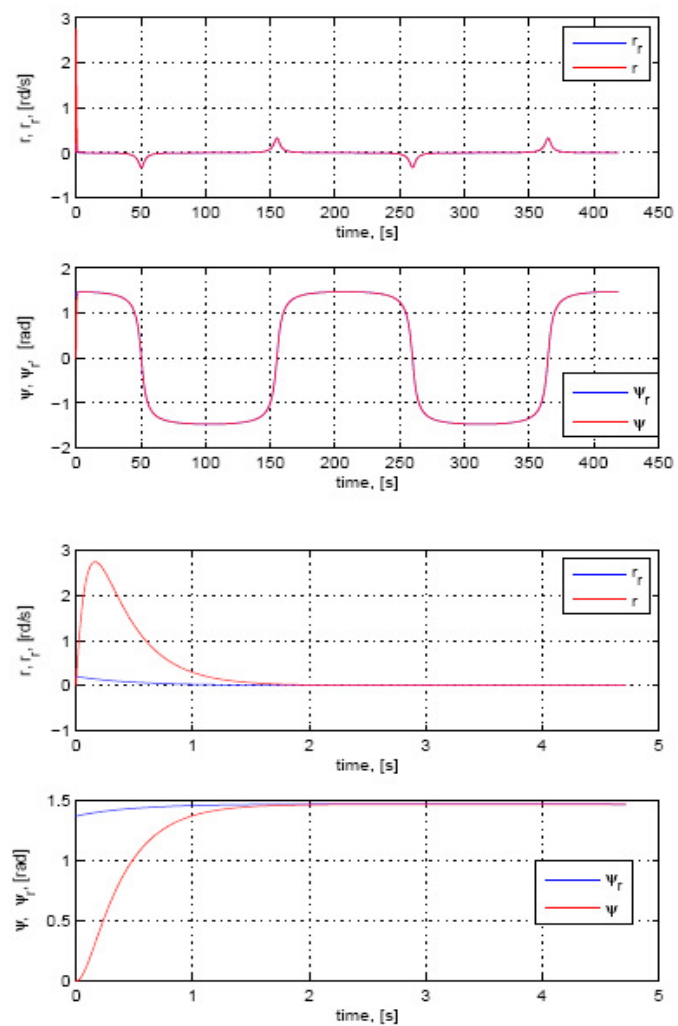

Fig. 12. (left side)Responses of $\tau_{u}$ and $\tau_{r}$ together with $\tau_{u_{r}}$ and $\tau_{r_{r}}$
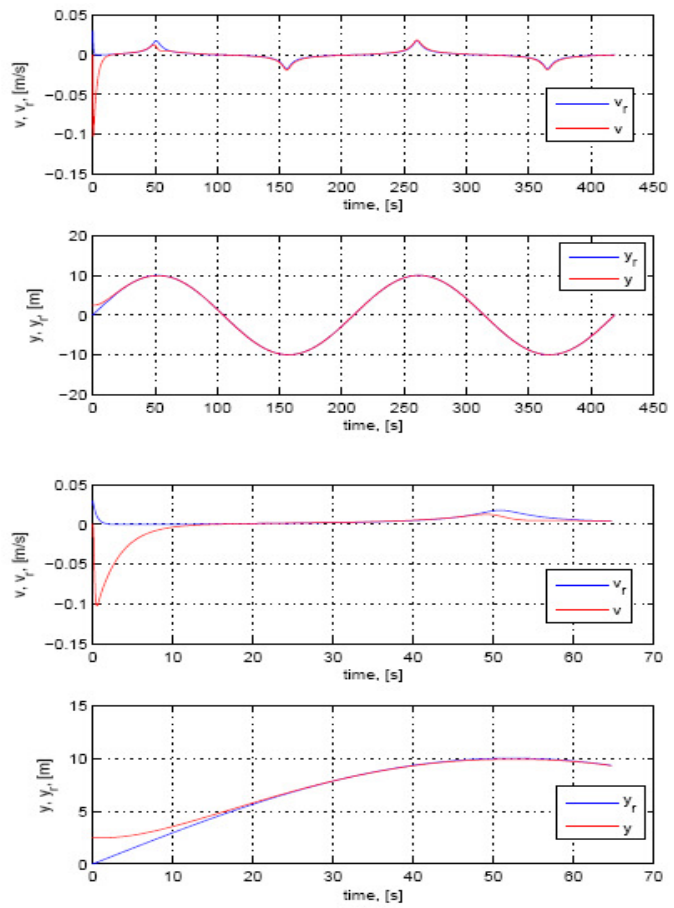

Fig. 9. (right side)Time responses of $x$ and $u$ together with $x_{r}$ and $u_{r}$
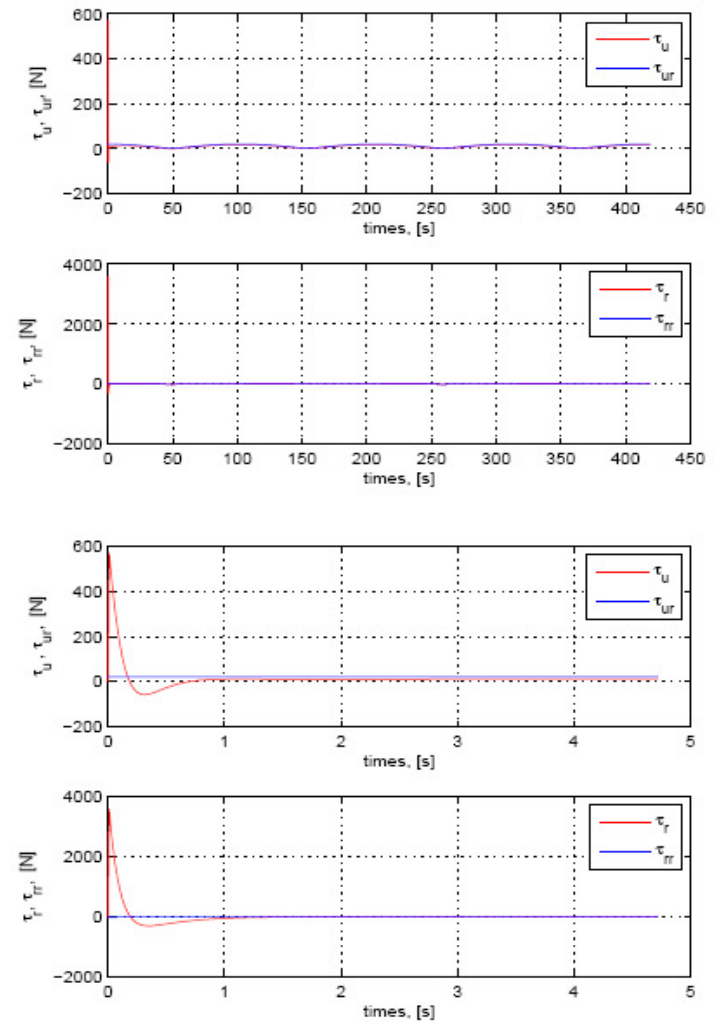

Fig. 11. (right side) Responses of $\psi$ and $r$ together with $\psi_{r}$ and $r_{r}$ 


\section{CONCLUSION}

In this paper, we presented an autonomous navigation control technique for an under-actuated ship. A Backstepping methodology combined with Lyapunov function for determining explicit stabilizing feedbacks, for the completed error system that assure the motion of the ship to track realisable trajectories, have been given. Simulation results show a very good tracking performance and effective robustness against considered disturbances.

\section{REFERENCES}

[1] Do, K. D. and Pan,J., "Global Tracking Control of Underactuated Ships with Nonzero Off-diagonal Terms in their System Matrices", Automatica, vol 41, pp 87-95 (2005).

[2] Repoulias, F. and Papadopoulos, E., Planar "Trajectory Planning Aerial vehicle stabilization and Tracking Control Design for Underactuated AUVs", Ocean Engineering, Vol 34, pp 1650-1667, (2007).

[3] Santhakumar, M. and Asokan, T., "Planar Tracking Control of an Underactuated Autonomous Underwater Vehicle" Int. Journal of Mechanical, Industrial and Aerospace Engineering, (2010).

[4] Lefeber, E., Pettersen, K.Y., Nijmeijer, H., "Tracking control of an underactuated Ship". IEEE Transactions on Control Systems Technology 11, 5261, (2003).

[5] Pettersen, K.Y. and Egeland, O., "Exponential Stabilization of an Underactuated Surface Vessel", in Proc. 35th IEEE Conference on Decision and Control, Kobe, Japan, pp. 967-971. DOI: 10.1109/CDC.1996.574602, (1996).

[6] Pettersen, K.Y., and Nijmeijer, H., "Tracking Control of an Underactuated Surface Vessel", in Proc. 37th IEEE Conference on Decision and Control, Tampa, Florida, pp. 4561-4566. DOI: 10.1109/CDC.1998.762046, (1998).

[7] Pettersen, K.Y., and Nijmeijer, H., "Underactuated ship tracking control: Theory and experiments", Inter. Journal of Control, vol. 74, No. 14, pp.14351446, (2001).

[8] Do, K. D. Jiang, P. and Pan,J., "Underactuated Ship Global Tracking Under Relaxed Conditions", IEEE Trans. Automat. Contr., vol 47, pp 1529-1536, (2002).

[9] Krstić, M.,Kanellakopoulos, I., Kokotovic, P., Non Linear and Adaptive Control Design, Wiley, NewYork (1995).

[10] Ghommam, J., Commande Nonlinéaire et Navigation des Véhicules Marins Sous-actionnés, Phd from University d'Orleans France, (2008).

[11] Khallil, H. K., Nonlinear Systems, second ed. Prentice-Hall, Upper Saddle River (1996).

[12] Dong, W. and Guo, P., "Nonlinear Tracking Control of Underactuated Surface Vessel", American Control Conference June 8-10. Portland, OR, USA (2005). 